\title{
A prospective study of physician-observed concussion during a varsity university hockey season: metabolic changes in ice hockey players. Part 4 of 4
}

\author{
Emilie Chamard, B.Sc., ${ }^{1}$ Hugo Théoret, Ph.D., ${ }^{1}$ Elaine N. Skopelja, M.A.L.S., ${ }^{2}$ \\ Lorie A. Forwell, M.Sc.P.T., ${ }^{3}$ Andrew M. Johnson, Ph.D., ${ }^{4}$ and Paul S. Echlin, M.D. 5 \\ ${ }^{1}$ Département de Psychologie, Université de Montréal and CHU Sainte-Justine, Montréal, Québec, Canada; \\ ${ }^{2}$ School of Medicine Library, Indiana University, Indianapolis, Indiana; ${ }^{3}$ Fowler Kennedy Sport Medicine \\ Clinic and School of Physical Therapy, The University of Western Ontario, London, Ontario; ${ }^{4}$ School of \\ Health Studies, The University of Western Ontario, London, Ontario; and ${ }^{5}$ Elliott Sports Medicine Clinic, \\ Burlington, Ontario, Canada
}

Object. Despite negative neuroimaging findings using traditional neuroimaging methods such as MRI and CT, sports-related concussions have been shown to cause neurometabolic changes in both the acute and subacute phases of head injury. However, no prospective clinical study has used an independent physician-observer design in the monitoring of these changes. The objective of this study was to evaluate the effects of repetitive concussive and subconcussive head impacts on neurometabolic concentrations in a prospective study of two Canadian Interuniversity Sports (CIS) ice hockey teams using MR spectroscopy (MRS).

Methods. Forty-five ice hockey players (25 men and 20 women) participated in this study. All participants underwent pre- and postseason MRI, including spectroscopy imaging, using a 3-T MRI machine. The linear combination model was used to quantify the following ratios: glutamate/creatine-phosphocreatine $(\mathrm{Cr})$, myoinositol/Cr, and $\mathrm{N}$-acetylaspartate (NAA)/Cr. Individuals sustaining a medically diagnosed concussion were sent for MRI at 72 hours, 2 weeks, and 2 months after injury.

Results. No statistically significant differences were observed between athletes who were diagnosed with a concussion and athletes who were not clinically diagnosed as sustaining a concussion. Although no statistically significant longitudinal metabolic changes were observed among athletes who were diagnosed with a concussion, the results demonstrated a predictable pattern of initial impairment, followed by a gradual return to ratios that were similar to, but lower than, baseline ratios. No significant pre- to postseason changes were demonstrated among men who were not observed to sustain a concussion. However, a substantively significant decrease in the NAA/Cr ratio was noted among the female hockey players $\left(t_{(13)}=2.58, p=0.02, \eta^{2}=0.34\right)$.

Conclusions. A key finding in this study, from the standpoint of future research design, is the demonstration of substantively significant metabolic changes among the players who were not diagnosed with a concussion. In addition, it may explain why there are few statistically significant differences demonstrated between players who were diagnosed with a concussion and players who were not diagnosed with a concussion (that is, the potency of the independent variable was diminished by the fact that the group of players not diagnosed with a concussion might be better described as a subgroup of the players who may have sustained a concussion but were not observed and diagnosed with a concussion). This result suggests that definitions of concussion may need to be revisited within sports with high levels of repetitive subconcussive head impacts. Future analysis of these data will examine the relationships between the modes of MRI (diffusion tensor imaging, MRS, and susceptibility-weighted MR imaging) used in this study, along with other more sensitive evaluative techniques. This type of intermodal comparison may improve the identification of concussions that were previously dependent on the unreliable self-reporting of recognized concussion symptomatology by the athlete or on poorly validated neuropsychological tests. (http://thejns.org/doi/abs/10.3171/2012.10.FOCUS12305)

$\begin{array}{llcl}\text { KEY WORDS } & \text { concussion } & \text { ice hockey } & \bullet \text { magnetic resonance imaging } \\ \text { magnetic resonance spectroscopy } & \bullet & \text { sex } & \bullet\end{array}$

\footnotetext{
Abbreviations used in this paper: CIS $=$ Canadian Interuniveristy Sports; $\mathrm{Cr}=$ creatine/phosphocreatine; DTI = diffusion tensor imaging; Glu = glutamate; HCEP = Hockey Concussion Education Project; $\mathrm{mI}=$ myoinositol; MRS = magnetic resonance spectroscopy; NAA $=N$-acetylaspartate; $\mathrm{SWI}=$ susceptibility-weighted $\mathrm{MR}$ imaging.
}

PORTS-RElated concussion is defined as a complex pathophysiological process affecting the brain in$\checkmark$ duced by traumatic biomechanical forces. ${ }^{22}$ This injury represents a major public health concern, as the annual prevalence of concussion is estimated to be between 1.6 and 3.8 million individuals in the US alone. ${ }^{18}$ 


\section{E. Chamard et al.}

However, concussion incidence appears to vary depending on the sport, patient sex, patient age, level of play, and type of exposure (such as time played per athlete, or number of games played per athlete). ${ }^{19}$

Ice hockey is a contact sport that has several inherent features that predispose players to brain injury. Typically, a greater proportion of concussions in ice hockey are reported in men than in women, ${ }^{19}$ with incidence rates reported to be as high as 21.5 concussions per 1000 athlete exposures. ${ }^{10}$ The results of the Injury Surveillance Study reported by the NCAA demonstrate that women's ice hockey has the second highest rate of concussions of all sports surveyed and the highest rate of concussions among the sports played by both sexes. ${ }^{1,15}$

Interestingly, although female ice hockey players report higher incidence rates of concussion than male players, ${ }^{1}$ the impact exposures in women are less frequent and of a lower magnitude. ${ }^{3}$ Note that although incidence rate may be useful for the description of player risk, its utility is heavily dependent on factors such as diagnosis criteria for concussion, observation method (whether the games are directly observed or whether the concussions are self-reported), and even observer type (physician vs nonphysician, or independent observer vs team-affiliated observer). ${ }^{8}$ Improved concussion screening-ideally in the form of an objectively scored, easily administered physiological measure-would provide a critical source of diagnostic evidence to the first responder that would be useful for medical specialists who are involved in the diagnosis and treatment of sport concussion. Such a screening test would also be useful for determining the most appropriate timeline for return to play, if it were sensitive to important individual differences in sensitivity to brain injury. However, such a test has not yet been validated for this purpose.

The physical, emotional, and cognitive symptoms following a concussion typically resolve after a period of 7-10 days in adults, ${ }^{20-22}$ a time window that closely coincides with the resolution of the neurometabolic cascade following a concussion. ${ }^{11}$ These symptoms are transient in the majority of observed cases, but appear in conjunction with the absence of the tissue damage that is visible using standard neuroimaging techniques such as CT and MRI. Studies of individuals who have sustained multiple sport concussions in their past have demonstrated objective evidence of chronic cognitive deficits and disorders. ${ }^{6,12,24,29}$ These data emphasize the importance of using the full spectrum of neuroimaging techniques to assess the potential brain alterations following a sport-related concussion.

Magnetic resonance spectroscopy is a neuroimaging technique that could be helpful in the investigation of brain alterations following a concussion, providing insight into the subtle neurophysiological alterations that accompany injury among concussed athletes. ${ }^{5}$ Magnetic resonance spectroscopy is a noninvasive technique that allows the detection and quantification of brain metabolites in the brain. Thus, it is a useful tool for detecting neuronal damage and diffuse axonal injury because damage-related changes to neurons are manifest not only in their physical structure, but also in their composition. ${ }^{28}$ The principal metabolites are NAA, a marker of neuronal and axonal integrity; $\mathrm{Cr}$, a fairly stable energy marker commonly used as an internal standard; $\mathrm{mI}$, an osmolyte and astrolyte marker; and Glu, a marker of excitatory neurotransmission.

An increasing number of studies of sports-related concussions have focused on brain metabolism changes in the acute phase following a concussion. Studies that assess the acute phase are particularly important ${ }^{13,14}$ because the athletes remain symptomatic during this time and experience a period of metabolic vulnerability within the brain tissue known as the "neurometabolic cascade" following a concussion. ${ }^{11}$ In the sports trauma literature, decreased levels of NAA have been found in the acute and subacute phases of concussion. ${ }^{13,14,16,17,30,31}$ However, the rate of recovery for this metabolite is still equivocal, with some studies findings NAA recovery to baseline levels within a period of 30-45 days after a concussion, ${ }^{31}$ and others demonstrating persistent decreases for at least 6 months after concussion. ${ }^{14}$ Moreover, the corpus callosum appears particularly vulnerable to neurometabolic disruptions and is a major predictor of predilection to concussion, ${ }^{26,27}$ being highly susceptible to the biomechanical forces induced by mild traumatic brain injury. ${ }^{25} \mathrm{~A}$ recent study has demonstrated lower NAA ratios in the genu and splenium of the corpus callosum in the subacute postinjury phase of concussed athletes. ${ }^{17}$ However, another recent study ${ }^{16}$ found a significant NAA decrease in the genu of the corpus callosum, but not in the splenium, in combination with a surprisingly greater alteration of the NAA ratio among patients recovering from their first concussion, as compared with athletes sustaining multiple concussions.

Despite recent studies assessing the neurometabolic profile of concussed athletes, few studies have examined the recovery pattern of metabolites following a concussion. Vagnozzi and colleagues ${ }^{30,31}$ evaluated athletes at a few time points after injury $(3,15,22$, and 30 days) and determined that concussions open a temporal window of metabolic imbalance. However, the control group was not composed of athletes, and the study population of the 2008 study contained both men and women with a sizeable age range, with no statistical treatment of sex differences. Moreover, this study ${ }^{31}$ focused exclusively on NAA recovery.

The current literature suggests that there is a need to assess the neurometabolic recovery of multiple metabolites in regions particularly vulnerable to brain injury, notably the corpus callosum, and to take into account possible sex differences for this recovery. Furthermore, it may be important to assess the extent to which there are metabolic changes among individuals with subclinical concussions or those who may have sustained multiple subconcussive blows to the head.

In this study, we sought to investigate the neurometabolic profiles of male and female ice hockey players following a sport-related concussion. To assess changes on the chemical structure of the corpus callosum over time, this study evaluated the concentration of NAA, mI, and Glu relative to $\mathrm{Cr}$ during the acute (72 hours) and subacute (2 weeks and 2 months) phases following a concussion. While no differences between groups are suspected at baseline, a significant decrease in NAA/Cr and Glu/ $\mathrm{Cr}$, as well as a significant increase in $\mathrm{mI} / \mathrm{Cr}$, was hypoth- 
esized to occur when athletes sustained clinically diagnosed concussions. Furthermore, a sequential recovery of the 3 metabolites was expected for group of individuals diagnosed with a concussion for each postinjury time point, with ratios approaching (but not reaching) baseline levels. Finally, it was expected that athletes who had not been diagnosed as sustaining a clinical concussion would demonstrate changes in their metabolite ratios over the course of the season due to multiple nonobserved injuries to the brain occurring as a result of regular play.

\section{Methods}

\section{Participants and Study Protocol}

Forty-five ice hockey players (25 men and 20 women) were included in this study. The male players were between 20 and 26 years of age (mean 22.24 years) and the female players were between 18.5 and 37.2 years of age (mean 20.21 years). All participants were part of the HCEP, a cohort study performed during a CIS ice hockey season (2011-2012). The clinical data for this study are described in detail by Echlin et al. ${ }^{9}$

Individuals were excluded from participation in this study if they had a history of central neurological conditions other than head injury, severe cognitive impairment (and/or an inability to consent), a history of pacemaker usage, previous eye surgery, or if they had worked in an environment that exposed them to a risk of having metal fragments embedded in their eyes. Each participant provided written informed consent and a release of medical information at the outset of the study. This study was approved by a university research ethics board.

This study focuses on the MRS analyses, whereas the detailed description and interpretation of concussion incidence and neuropsychological testing, as well as DTI results are presented in other papers within this issue of Neurosurgical Focus. Susceptibility-weighted MR imaging results from this study will be published in the near future.

\section{Magnetic Resonance Imaging Protocol and Data Acquisition}

Data acquisition was performed using a 3-T MRI machine (Achieva, Phillips) equipped with an 8-channel SENSE head coil array. Each player involved in this study received a baseline MRI evaluation. Athletes sustaining a concussion underwent further imaging at 72 hours, 2 weeks, and 2 months postinjury.

\section{Magnetic Resonance Spectroscopy}

The corpus callosum was chosen as the MRS region of interest because it forms the largest and highest-density commissural white matter bundle in the brain. It also provides interhemispheric connections that project to all cerebral lobes. The corpus callosum is suspected to be highly vulnerable to the biomechanical forces involved with concussion. 7,16,17,26,27,32 The placement of the voxel within the corpus callosum attempted to ensure an adequate distance from the ventricles, fatty tissue, and bone for all patients.

The cognitive demands of playing ice hockey include rapid and effective information transmission in the brain to allow adequate decision-making and execution of complex motor sequences. The examination of metabolite profiles within the corpus callosum may provide evidence of the effects of microstructural alterations within this structure and on transmission processes between the cerebral lobes.

Each patient was placed in the MRI machine and underwent a standard localizer and SENSE calibration scan. Voxels were then applied for the corpus callosum $(10 \times$ $20 \times 30 \mathrm{~mm})$. Spectroscopic examination was carried out using a PRESS (Point RESolved Spectroscopy) sequence pulse with the following settings: TE $35 \mathrm{msec}$, TR 2000 msec, 128 acquisitions, and 1024 points, on an 8-channel head coil.

The linear combination model ${ }^{23}$ was used for metabolite quantification (see Fig. 1 for a reference figure of metabolic analysis). This operator-independent spectral analysis software estimates metabolite concentrations and their ratios relative to $\mathrm{Cr}$ using a set of basis reference spectra acquired from individual metabolites on the MR instrument. Concentrations are derived from the areas under the corresponding peaks. $\mathrm{N}$-acetylaspartate/Cr, $\mathrm{Glu} / \mathrm{Cr}$, and $\mathrm{mI} / \mathrm{Cr}$ were only analyzed if the estimated uncertainty calculated as Cramer Ratio lower bounds (\% SD) was less than $20 \%$. The linear combination model operator was blind to group membership.

\section{Statistical Analysis}

Statistical analyses of metabolite ratios $(\mathrm{Glu} / \mathrm{Cr}, \mathrm{mI} / \mathrm{Cr}$, and NAA/Cr) were conducted using SPSS version 20. Due to the small number of concussed individuals in whom we had complete data across the postconcussion time periods, data analysis was performed graphically rather than statistically. Accordingly, the average metabolite ratios were

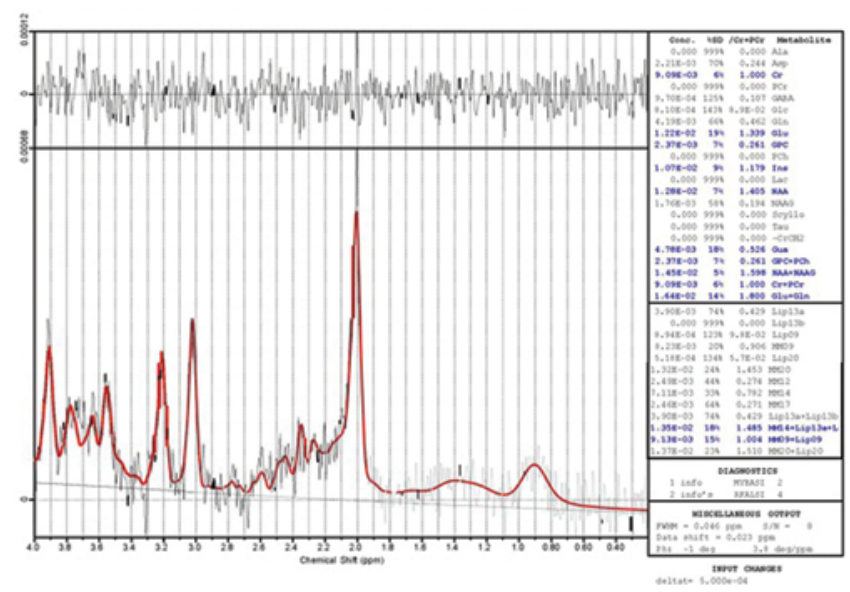

Fig. 1. An example of a metabolite spectrum produced by the linear combination model that was used for metabolite quantification. This operator-independent spectral analysis software estimates metabolite concentrations and their ratios relative to $\mathrm{Cr}$, using a set of basis reference spectra acquired from individual metabolites on the MR instrument. Concentrations are derived from the areas under the corresponding peaks. NAA/Cr, Glu/Cr, and $\mathrm{ml} / \mathrm{Cr}$ were only analyzed if the estimated uncertainty calculated as Cramer Ratio lower bounds (\% SD) was less than $20 \%$. The linear combination model operator was blind to group membership. 
plotted at each time point (baseline, 72 hours, 2 weeks, and 2 months), with error bars representing \pm 1 standard error. Individuals who were not diagnosed as having sustained a concussion were evaluated separately for each sex using a series of paired t-tests. Adjustments to the comparison $\alpha$ were performed within each sex using a Bonferroni adjustment; to maintain an overall experiment-wise $\alpha$ of 0.05 , each of the 3 comparisons conducted within each sex was made against an $\alpha$ of $0.017(0.05 / 3)$. Finally, a split-plot ANOVA was used to determine the extent to which group membership (individuals diagnosed with a concussion vs those not diagnosed with a concussion) interacted with time course (preseason vs postseason) in predicting each of the 3 metabolite ratios.

\section{Results}

Descriptive statistics for each metabolite ratio at baseline assessment and at each of the 3 postinjury evaluations are presented in Table 1 and depicted graphically in Fig. 2.

Descriptive statistics for the individuals within the sample who were not clinically identified as sustaining a concussion are presented in Table 2 along with the results of the paired t-test calculations. Although none of the t-tests were statistically significant after adjusting for potential inflation of a Type I error due to multiple comparison bias, women appeared to demonstrate a substantively significant decrease in their NAA/Cr ratios over the course of the season $\left(\mathrm{t}_{(13)}=2.58, \mathrm{p}=0.02, \eta^{2}=0.34\right)$ that suggests that $34 \%$ of the postseason variability on this metabolite was due to subconcussive impacts sustained over the course of the season.

No statistically significant effects were demonstrated for the interaction between group and time for Glu/ $\mathrm{Cr}\left(\mathrm{F}_{(1,31)}=2.945, \mathrm{p}=0.096\right), \mathrm{mI} / \mathrm{Cr}\left(\mathrm{F}_{(1,31)}=0.820, \mathrm{p}=\right.$ $0.372)$, or NAA/Cr $\left(\mathrm{F}_{(1,31)}=0.214, \mathrm{p}=0.646\right)$, suggesting that seasonal changes did not differ significantly between individuals who were diagnosed with a concussion and individuals who were not diagnosed with a concussion.

\section{Discussion}

The present study is unique in that it is a part of a multidimensional prospective effort (the HCEP) to independently and directly evaluate sport concussion incidence as well as the neuropsychological and multimodal MRI changes in the participant group over 1 season of CIS varsity hockey. The aim of the present study was to investigate the neurometabolic alterations in the corpus callosum of male and female ice hockey players following a concussion. A second aim of this study was to investigate the metabolite changes of athletes who had not been diagnosed with a concussion over the pre- to postseason time interval. Using MRS, no statistically significant differences were found for any metabolite ratios when comparing athletes diagnosed with a concussion to control athletes.

The most interesting findings within this study were found in female athletes within the group of individuals who were not diagnosed with a concussion. This group demonstrated a decrease in their NAA/Cr ratio over the course of the season. The ratio of each metabolite remained stable in the sample of male athletes who were not diagnosed with a concussion.

The results of the present study do not corroborate the findings of previous researchers, who demonstrated a decrease in the NAA ratio within the acute phase of a concussion..$^{13,14,31}$ Several factors could explain the different metabolic profiles found in this study. First, previous studies investigated different regions of interest, notably the frontal regions, the primary motor cortices, and different regions within the corpus callosum where neurometabolic changes were different depending on the regions observed..$^{13,14}$ The different metabolites in any given voxel are, in principle, unrelated and uncorrelated. ${ }^{4}$ Based on this assumption, it is possible that the neurometabolic alterations observed in previous studies were specific to the cerebral regions examined in those studies and the corpus callosum may not be affected in the acute phase of a concussion, as demonstrated in the current study. It is possible that there were no effects of concussions on metabolite concentration in the present sample because of the anatomical variation of voxel location. Future studies should examine cortical and subcortical regions following a brain injury to determine the pattern of changes in neurochemistry specific to each region.

Second, the sample in the present study differed from samples used in previous studies. The sample in the study of Vagnozzi et al. ${ }^{31}$ was composed of a large age range of men and women from different sports (kickboxing, boxing, soccer, alpine skiing, and rugby), while the studies of Henry et al. ${ }^{13,14}$ included only male football players. The heterogeneity of the group who was diagnosed with a concussion combined with a control group composed of nonathletes in the study of Vagnozzi et al. ${ }^{31}$ makes it difficult to compare their findings with the results of the present study. Furthermore, the present study found that

TABLE 1: Descriptive statistics for individuals sustaining a clinically diagnosed concussion at baseline and at 3 time points after injury

\begin{tabular}{|c|c|c|c|c|c|c|c|c|c|}
\hline \multirow[b]{2}{*}{ Evaluation Period } & \multicolumn{3}{|c|}{$\mathrm{Glu} / \mathrm{Cr}$} & \multicolumn{3}{|c|}{$\mathrm{ml} / \mathrm{Cr}$} & \multicolumn{3}{|c|}{$\mathrm{NAA} / \mathrm{Cr}$} \\
\hline & Mean & SD & $\begin{array}{c}\text { No. of } \\
\text { Patients }\end{array}$ & Mean & SD & $\begin{array}{l}\text { No. of } \\
\text { Patients }\end{array}$ & Mean & SD & $\begin{array}{l}\text { No. of } \\
\text { Patients }\end{array}$ \\
\hline baseline & 1.75 & 0.23 & 11 & 0.97 & 0.28 & 11 & 1.66 & 0.30 & 11 \\
\hline 72 hrs postinjury & 1.71 & 0.10 & 7 & 0.93 & 0.18 & 8 & 1.64 & 0.19 & 8 \\
\hline 2 wks postinjury & 1.79 & 0.20 & 10 & 0.91 & 0.13 & 10 & 1.65 & 0.36 & 10 \\
\hline 2 mos postinjury & 1.69 & 0.26 & 9 & 0.99 & 0.11 & 9 & 1.63 & 0.14 & 9 \\
\hline
\end{tabular}




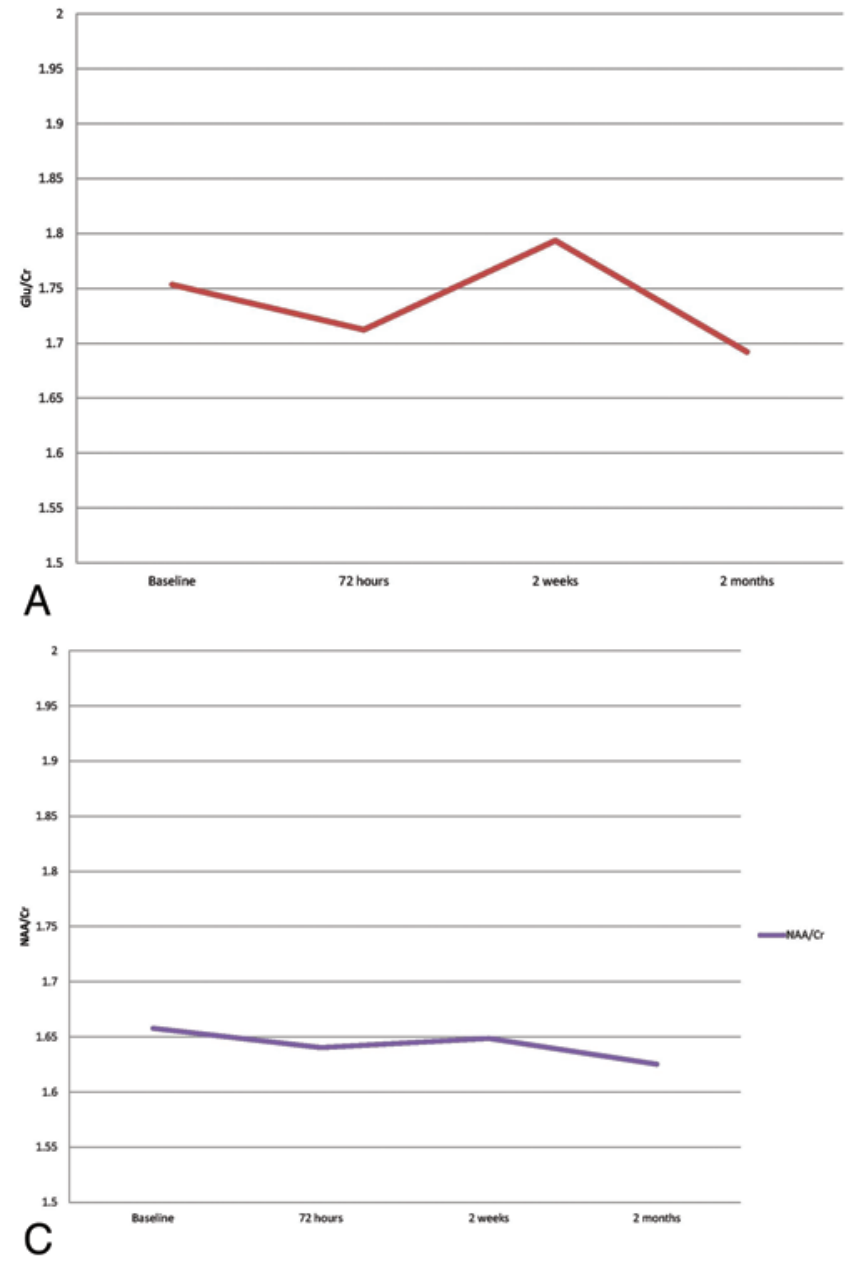

there were substantively significant differences between preseason and postseason NAA/Cr ratios among the female players. These data suggest that the primary independent variable - the group who was diagnosed with a concussion versus the group who was not diagnosed with a concussion-was of reduced potency.

An interesting finding in the present study is the difference between male and female athletes over the course of the season, and a key variable appears to be NAA/ $\mathrm{Cr}$. While relative quantification of metabolite ratios remained stable over the course of 1 season in male athletes, female athletes showed a decrease in NAA ratios. A recent study demonstrated that female hockey players sustain fewer impacts and suggested that the impacts in which they are involved result in lower head acceleration compared with male athletes. ${ }^{3}$ However, female athletes have a higher incidence of concussion than their male counterparts, ${ }^{1}$ possibly reflecting a higher sensitivity to head impacts and subconcussive blows sustained during practices and games over the course of 1 season. This provisional hypothesis is corroborated by a recent DTI study examining multiple subconcussive blows. ${ }^{2}$ The sex differences shown in the present research emphasize the importance of assessing the cerebral alterations specific to each sex following a brain injury, as this will allow

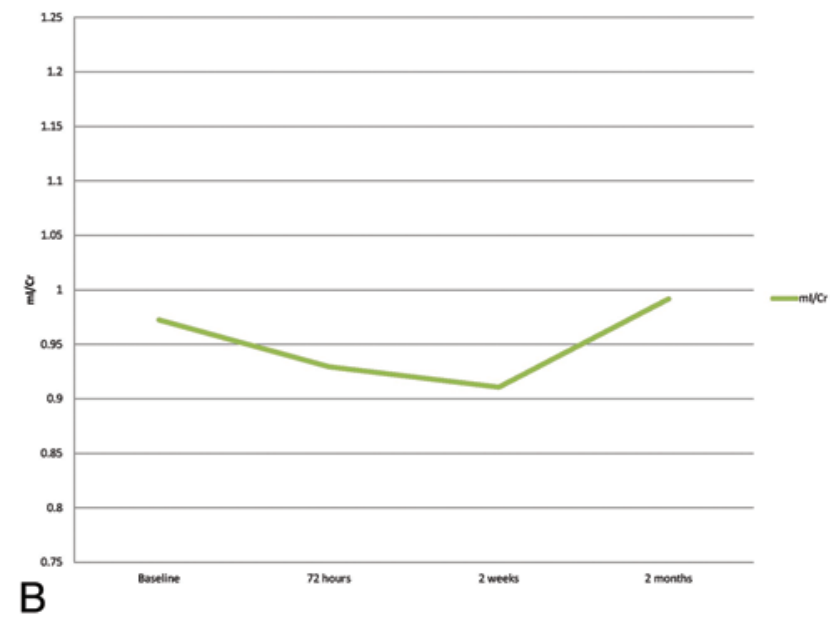

FIG. 2. The temporal concentration of Glu relative to $\mathrm{Cr}(\mathrm{A})$, $\mathrm{ml}$ relative to $\mathrm{Cr}(\mathrm{B})$, and NAA relative to $\mathrm{Cr}(\mathrm{C})$ during the acute (72 hours) and subacute ( 2 weeks and 2 months) phases following a concussion.

us to better understand the pathological mechanisms involved in this injury, and ultimately, to ensure a safe return to play for these athletes.

An important limitation of this study, common to most neuroimaging studies, is the small sample size. The small number of participants of both sexes in each group limits the extent to which the current findings can be generalized to all sports practiced by both sexes. The study also lacks a control group of matched participant athletes from a collegiate varsity sport with a low probability of head contact (such as swimming). The latter change is likely to result in an independent variable (group difference) of higher potency and would increase the power of the study.

\section{Conclusions}

Physician observation and diagnosis using current international diagnostic criteria offers a unique design to assess neurochemical changes in the acute and subacute phases of concussion in both men and women ice hockey players.

The nonsignificant differences in metabolic ratios between those players who were clinically diagnosed with a concussion and those individuals who were not identi- 
E. Chamard et al.

TABLE 2: Descriptive statistics for individuals not clinically identified as sustaining a concussion over the course of the ice hockey season*

\begin{tabular}{|c|c|c|c|c|c|}
\hline \multirow[b]{2}{*}{ Variable } & \multirow[b]{2}{*}{ Baseline } & \multirow[b]{2}{*}{ End of Season } & \multicolumn{3}{|c|}{ t-Test } \\
\hline & & & $t_{\text {(df) }}$ Value & $p$ Value & $\eta^{2} \dagger$ \\
\hline \multicolumn{6}{|l|}{ men } \\
\hline $\mathrm{Glu} / \mathrm{Cr}$ & & & $t_{(10)}=0.56$ & 0.59 & 0.03 \\
\hline mean & 1.81 & 1.76 & & & \\
\hline SD & 0.30 & 0.24 & & & \\
\hline no. of patients & 11 & 11 & & & \\
\hline $\mathrm{ml} / \mathrm{Cr}$ & & & $t_{(13)}=0.027$ & 0.98 & 0.00 \\
\hline mean & 0.98 & 0.97 & & & \\
\hline SD & 0.23 & 0.21 & & & \\
\hline no. of patients & 14 & 14 & & & \\
\hline $\mathrm{NAA} / \mathrm{Cr}$ & & & $t_{(15)}=0.44$ & 0.97 & 0.01 \\
\hline mean & 1.62 & 1.63 & & & \\
\hline SD & 0.23 & 0.26 & & & \\
\hline no. of patients & 16 & 16 & & & \\
\hline \multicolumn{6}{|l|}{ women } \\
\hline $\mathrm{Glu} / \mathrm{Cr}$ & & & $t_{(13)}=0.46$ & 0.66 & 0.02 \\
\hline mean & 1.93 & 1.90 & & & \\
\hline $\mathrm{SD}$ & 0.20 & 0.26 & & & \\
\hline no. of patients & 14 & 14 & & & \\
\hline $\mathrm{ml} / \mathrm{Cr}$ & & & $t_{(12)}=0.28$ & 0.79 & 0.01 \\
\hline mean & 0.97 & 0.98 & & & \\
\hline SD & 0.13 & 0.17 & & & \\
\hline no. of patients & 13 & 13 & & & \\
\hline $\mathrm{NAA} / \mathrm{Cr}$ & & & $t_{(13)}=2.58$ & 0.02 & 0.34 \\
\hline mean & 1.90 & 1.69 & & & \\
\hline SD & 0.32 & 0.27 & & & \\
\hline no. of patients & 14 & 14 & & & \\
\hline
\end{tabular}

fied as sustaining a concussion may be interpreted as evidence of nondiagnosed (and nonobserved) subconcussive neural injuries sustained over a regular season of play. Future analysis of these data will examine the relationships between the modes of MRI (DTI, MRS, and SWI) used in this study, along with other more sensitive evaluative techniques. This type of intermodal comparison may improve the identification of concussions that were previously dependent upon unreliable self-reported concussion symptoms by the athlete, supplemented by poorly validated neuropsychological tests.

This unique and multidimensional prospective study demonstrates a pressing need for follow-up studies using a similar design, involving independent physician and nonphysican observers, within simultaneous multimodal objective investigation.

The potential consequences of sustaining both clinically diagnosed concussions and nondiagnosed recurrent subconcussive blows should be explored temporally. Follow-up studies should also consider further investigation of sex differences in neurochemistry. In future publications a comparison of the neurochemical changes among subjects should be made to the other objective investigations (such as DTI and SWI) that were simultaneously used. The involvement of larger and more diverse sample groups will allow for a greater generalizability of the findings.

\section{Disclosure}

Funding for this work was provided to the HCEP and Dr. Echlin by the Ontario Trillium Foundation, the Dave Irwin Foundation for Brain Injury, the Ontario Neurotrauma Foundation, and Air Canada.

Author contributions to the study and manuscript preparation include the following. Conception and design: all authors. Acquisition of data: Echlin, Forwell. Analysis and interpretation of data: all authors. Drafting the article: Echlin, Chamard, Skopelja, Forwell, Johnson. Critically revising the article: all authors. Reviewed submitted version of manuscript: all authors. Approved the final version of the manuscript on behalf of all authors: Echlin. Statistical analysis: Echlin, Chamard, Johnson. Administrative/technical/material support: Echlin, Skopelja, Forwell. Study supervision: Theoret, Forwell. 


\section{Acknowledgments}

The authors would like to acknowledge the players and staffs of two CIS varsity hockey teams for their participation in the HCEP, and would also like to acknowledge the participating physicians, observers, and volunteers for their contributions to the HCEP.

\section{References}

1. Agel J, Dick R, Nelson B, Marshall SW, Dompier TP: Descriptive epidemiology of collegiate women's ice hockey injuries: National Collegiate Athletic Association Injury Surveillance System, 2000-2001 through 2003-2004. J Athl Train 42:249-254, 2007

2. Bazarian JJ, Zhu T, Blyth B, Borrino A, Zhong J: Subjectspecific changes in brain white matter on diffusion tensor imaging after sports-related concussion. Magn Reson Imaging 30:171-180, 2012

3. Brainard LL, Beckwith JG, Chu JJ, Crisco JJ, McAllister TW, Duhaime AC, et al: Gender differences in head impacts sustained by collegiate ice hockey players. Med Sci Sports Exerc 44:297-304, 2012

4. Braun CM, Boulanger Y, Labelle M, Khiat A, Dumont M, Mailloux C: Brain metabolic differences as a function of hemisphere, writing hand preference, and gender. Laterality 7: 97-113, 2002

5. De Beaumont L, Lassonde M, Leclerc S, Théoret H: Longterm and cumulative effects of sports concussion on motor cortex inhibition. Neurosurgery 61:329-337, 2007

6. De Beaumont L, Théoret H, Mongeon D, Messier J, Leclerc $\mathrm{S}$, Tremblay $\mathrm{S}$, et al: Brain function decline in healthy retired athletes who sustained their last sports concussion in early adulthood. Brain 132:695-708, 2009

7. Ducreux D, Huynh I, Fillard P, Renoux J, Petit-Lacour MC, Marsot-Dupuch K, et al: Brain MR diffusion tensor imaging and fibre tracking to differentiate between two diffuse axonal injuries. Neuroradiology 47:604-608, 2005

8. Echlin PS: Concussion education, identification, and treatment within a prospective study of physician-observed junior ice hockey concussions: social context of this scientific intervention. Neurosurg Focus 29(5):E7, 2010

9. Echlin PS, Skopelja EN, Worsley R, Dadachanji SB, LloydSmith DR, Taunton JA, et al: A prospective study of physician-observed concussion during a varsity university ice hockey season: incidence and neuropsychological changes. Part 2 of 4. Neurosurg Focus 33(6):E2, 2012

10. Echlin PS, Tator CH, Cusimano MD, Cantu RC, Taunton JE, Upshur RE, et al: A prospective study of physician-observed concussions during junior ice hockey: implications for incidence rates. Neurosurg Focus 29(5):E4, 2010

11. Giza CC, Hovda DA: The neurometabolic cascade of concussion. J Athl Train 36:228-235, 2001

12. Guskiewicz KM, Marshall SW, Bailes J, McCrea M, Cantu $\mathrm{RC}$, Randolph C, et al: Association between recurrent concussion and late-life cognitive impairment in retired professional football players. Neurosurgery 57:719-726, 2005

13. Henry LC, Tremblay S, Boulanger Y, Ellemberg D, Lassonde M: Neurometabolic changes in the acute phase after sports concussions correlate with symptom severity. J Neurotrauma 27:65-76, 2010

14. Henry LC, Tremblay S, Leclerc S, Khiat A, Boulanger Y, Ellemberg D, et al: Metabolic changes in concussed American football players during the acute and chronic post-injury phases. BMC Neurol 11:105, 2011

15. Hootman JM, Dick R, Agel J: Epidemiology of collegiate injuries for 15 sports: summary and recommendations for injury prevention initiatives. J Athl Train 42:311-319, 2007

16. Johnson B, Gay M, Zhang K, Neuberger T, Horovitz SG, Hallett $\mathrm{M}$, et al: The use of magnetic resonance spectroscopy in the subacute evaluation of athletes recovering from single and multiple mild traumatic brain injury. J Neurotrauma 29: 2297-2304, 2012

17. Johnson B, Zhang K, Gay M, Neuberger T, Horovitz S, Hallett $\mathrm{M}$, et al: Metabolic alterations in corpus callosum may compromise brain functional connectivity in MTBI patients: an 1H-MRS study. Neurosci Lett 509:5-8, 2012

18. Langlois JA, Rutland-Brown W, Wald MM: The epidemiology and impact of traumatic brain injury: a brief overview. J Head Trauma Rehabil 21:375-378, 2006

19. Marar M, McIlvain NM, Fields SK, Comstock RD: Epidemiology of concussions among United States high school athletes in 20 sports. Am J Sports Med 40:747-755, 2012

20. McCrea M, Guskiewicz KM, Marshall SW, Barr W, Randolph C, Cantu RC, et al: Acute effects and recovery time following concussion in collegiate football players: the NCAA Concussion Study. JAMA 290:2556-2563, 2003

21. McCrory P, Johnston K, Meeuwisse W, Aubry M, Cantu R, Dvorak J, et al: Summary and agreement statement of the 2nd International Conference on Concussion in Sport, Prague 2004. Br J Sports Med 39:196-204, 2005

22. McCrory P, Meeuwisse W, Johnston K, Dvorak J, Aubry M, Molloy M, et al: Consensus statement on Concussion in Sport 3rd International Conference on Concussion in Sport held in Zurich, November 2008. Clin J Sport Med 19:185-200, 2009

23. Provencher SW: Estimation of metabolite concentrations from localized in vivo proton NMR spectra. Magn Reson Med 30:672-679, 1993

24. Rabadi MH, Jordan BD: The cumulative effect of repetitive concussion in sports. Clin J Sport Med 11:194-198, 2001

25. Rutgers DR, Fillard P, Paradot G, Tadié M, Lasjaunias P, Ducreux D: Diffusion tensor imaging characteristics of the corpus callosum in mild, moderate, and severe traumatic brain injury. AJNR Am J Neuroradiol 29:1730-1735, 2008

26. Smits M, Houston GC, Dippel DW, Wielopolski PA, Vernooij MW, Koudstaal PJ, et al: Microstructural brain injury in postconcussion syndrome after minor head injury. Neuroradiology 53:553-563, 2011

27. Sponheim SR, McGuire KA, Kang SS, Davenport ND, Aviyente $\mathrm{S}$, Bernat EM, et al: Evidence of disrupted functional connectivity in the brain after combat-related blast injury. Neuroimage 54 (Suppl 1):S21-S29, 2011

28. Toga AW, Mazziotta JC: Brain Mapping: The Methods, ed 2. San Diego, CA: Academic Press, 2002

29. Tremblay S, De Beaumont L, Henry LC, Boulanger Y, Evans $\mathrm{AC}$, Bourgouin $\mathrm{P}$, et al: Sports concussions and aging: a neuroimaging investigation. Cereb Cortex [epub ahead of print], 2012

30. Vagnozzi R, Signoretti S, Cristofori L, Alessandrini F, Floris $\mathrm{R}$, Isgrò $\mathrm{E}$, et al: Assessment of metabolic brain damage and recovery following mild traumatic brain injury: a multicentre, proton magnetic resonance spectroscopic study in concussed patients. Brain 133:3232-3242, 2010

31. Vagnozzi R, Signoretti S, Tavazzi B, Floris R, Ludovici A, Marziali S, et al: Temporal window of metabolic brain vulnerability to concussion: a pilot $1 \mathrm{H}$-magnetic resonance spectroscopic study in concussed athletes-part III. Neurosurgery 62:1286-1296, 2008

32. Wilde EA, McCauley SR, Hunter JV, Bigler ED, Chu Z, Wang $\mathrm{ZJ}$, et al: Diffusion tensor imaging of acute mild traumatic brain injury in adolescents. Neurology 70:948-955, 2008

Manuscript submitted September 17, 2012.

Accepted October 22, 2012.

Please include this information when citing this paper: DOI: 10.3171/2012.10.FOCUS12305.

Address correspondence to: Paul S. Echlin, M.D., Elliott Sports Medicine Clinic, 1100 Walkers Line, Suite 2, Burlington, Ontario, Canada L7N 2G3. email: psechlin@gmail.com. 